IJMMS 30:12 (2002) 727-731

PII. S0161171202109264

http://ijmms.hindawi.com

(c) Hindawi Publishing Corp.

\title{
EQUIVALENCE RESULTS FOR DISCRETE ABEL MEANS
}

\section{JEFFREY A. OSIKIEWICZ}

Received 7 September 2001

We present theorems showing when the discrete Abel mean and the Abel summability method are equivalent for bounded sequences and when two discrete Abel means are equivalent for bounded sequences.

2000 Mathematics Subject Classification: 40D25, 40G10.

1. Introduction and notation. The well-known Abel summability method is a sequence-to-function transformation which is defined as follows: for a sequence $s:=$ $\left\{s_{n}\right\}$ of complex numbers, define

$$
f(x):=(1-x) \sum_{k=0}^{\infty} s_{k} x^{k},
$$

for all $x$ for which the series converges. If $f(x)$ exists for each $x \in(0,1)$ and $\lim _{x \rightarrow 1^{-}} f(x)=L$, then the sequence $s$ is Abel summable to $L$. The discrete Abel mean is a sequence-to-sequence transformation given by the summability matrix $A_{\lambda}$ whose $n k$ th entry is

$$
A_{\lambda}[n, k]:=\frac{1}{\lambda(n)}\left(1-\frac{1}{\lambda(n)}\right)^{k}, \quad n, k=0,1,2,3, \ldots,
$$

where $\lambda:=\{\lambda(n)\}$ is a strictly increasing sequence of real numbers such that $\lambda(0) \geq 1$ and $\lambda(n) \rightarrow \infty$. Then the sequence $s$ is $A_{\lambda}$-summable to $L$ provided that

$$
\lim _{n \rightarrow \infty}\left(A_{\lambda} s\right)_{n}=\lim _{n \rightarrow \infty} \frac{1}{\lambda(n)} \sum_{k=0}^{\infty} s_{k}\left(1-\frac{1}{\lambda(n)}\right)^{k}=L .
$$

In [1], Armitage and Maddox proved inclusion and Tauberian theorems for the discrete Abel mean. In this paper, we expand upon the work of these authors by examining equivalence properties of the $A_{\lambda}$ method for bounded sequences.

For a given sequence $s$, define a sequence $a$ by $a_{0}:=s_{0}$ and $a_{n}:=s_{n}-s_{n-1}$ for $n \geq 1$. Then, $s_{n}=\sum_{k=0}^{n} a_{k}$ and for every $n$,

$$
\left(A_{\lambda} s\right)_{n}=\frac{1}{\lambda(n)} \sum_{k=0}^{\infty} s_{k}\left(1-\frac{1}{\lambda(n)}\right)^{k}=\sum_{k=0}^{\infty} a_{k}\left(1-\frac{1}{\lambda(n)}\right)^{k} .
$$

Also, define the sequence $t$ by

$$
t_{n}:=\sum_{k=1}^{n} k a_{k}
$$


A straightforward induction argument yields

$$
t_{n}=\sum_{k=0}^{n}\left(s_{n}-s_{k}\right)
$$

If $B$ and $C$ are two summability methods, then $C$ includes $B$, denoted $B \subset C$, provided that every sequence which is $B$-summable is also $C$-summable to the same limit. If $B \subset C$ and $C \subset B$, then $B$ and $C$ are equivalent, denoted $B \sim C$.

2. Equivalence results. For any sequence $\lambda, A_{\lambda}$ is clearly a regular (i.e., limit preserving) method. In [1], Armitage and Maddox proved the following inclusion results for the $A_{\lambda}$ method.

TheOrem 2.1 (see [1]). Let $E(\lambda):=\{\lambda(n): n=0,1,2, \ldots\}$ and $E(\mu):=\{\mu(n): n=$ $0,1,2, \ldots\}$. Then

(1) $A_{\lambda} \subset A_{\mu}$ if and only if $E(\mu) \backslash E(\lambda)$ is a finite set;

(2) $A_{\mu} \sim A_{\lambda}$ if and only if the symmetric difference $E(\lambda) \triangle E(\mu)$ is a finite set.

COROLlary 2.2 (see [1]). For every $\lambda, A_{\lambda}$ strictly includes the Abel method.

The main result of this section is that $A_{\lambda}$ is equivalent to the Abel method for bounded sequences provided that $\lambda(n+1) / \lambda(n) \rightarrow 1$. To show this we need the following two lemmas.

LEMMA 2.3 (see [1]). If $\sum_{k=0}^{\infty} a_{k} x^{k}$ converges for all $x \in(0,1)$, then

$$
\sum_{k=1}^{\infty} a_{k} x^{k}=\sum_{k=1}^{\infty} t_{k} \Delta\left(\frac{x^{k}}{k}\right), \quad 0<x<1,
$$

where $\Delta\left(x^{k} / k\right)=x^{k} / k-x^{k+1} /(k+1)$.

LEMMA 2.4. If $s$ is a bounded sequence, then $t_{n}=O(n)$.

Proof. Let $s$ be a bounded sequence. By (1.6),

$$
\begin{aligned}
\left|t_{n}\right|=\left|\sum_{k=0}^{n}\left(s_{n}-s_{k}\right)\right| & =\left|(n+1) s_{n}-\sum_{k=0}^{n} s_{k}\right| \\
& \leq(n+1)\|s\|_{\infty}+\sum_{k=0}^{n}\left|s_{k}\right| \\
& \leq(n+1)\|s\|_{\infty}+(n+1)\|s\|_{\infty} \\
& =O(n) .
\end{aligned}
$$

THEOREM 2.5. If $\lim _{n \rightarrow \infty}(\lambda(n+1) / \lambda(n))=1$, then $A_{\lambda}$ is equivalent to the Abel method for bounded sequences. 
Proof. By Corollary 2.2, $A_{\lambda}$ includes the Abel method. So assume that

$$
\lim _{n \rightarrow \infty}\left(\frac{\lambda(n+1)}{\lambda(n)}\right)=1
$$

let $s$ be a bounded sequence, that is, $A_{\lambda}$-summable to $L$, and let $a$ be the sequence such that $s_{n}=\sum_{k=0}^{n} a_{k}$. Let $x_{n}:=1-1 / \lambda(n)$. Then, for a given $x \in\left(x_{0}, 1\right)$, there exists an $n$ such that $x_{n}<x \leq x_{n+1}$. By (1.1) and (1.4),

$$
\begin{aligned}
\left|f(x)-\left(A_{\lambda} s\right)_{n}\right| & =\left|(1-x) \sum_{k=0}^{\infty} s_{k} x^{k}-\frac{1}{\lambda(n)} \sum_{k=0}^{\infty} s_{k}\left(1-\frac{1}{\lambda(n)}\right)^{k}\right| \\
& =\left|\sum_{k=0}^{\infty} a_{k} x^{k}-\sum_{k=0}^{\infty} a_{k} x_{n}{ }^{k}\right| .
\end{aligned}
$$

By Lemma 2.3, this becomes

$$
\begin{aligned}
\left|f(x)-\left(A_{\lambda} s\right)_{n}\right| & =\left|\sum_{k=1}^{\infty} t_{k} \Delta\left(\frac{x^{k}}{k}\right)-\sum_{k=1}^{\infty} t_{k} \Delta\left(\frac{x_{n}^{k}}{k}\right)\right| \\
& =\left|\sum_{k=1}^{\infty} t_{k} \int_{x_{n}}^{x} t^{k-1}(1-t) d t\right| \\
& \leq \sum_{k=1}^{\infty}\left|t_{k}\right| \int_{x_{n}}^{x_{n+1}} t^{k-1}(1-t) d t
\end{aligned}
$$

By Lemma 2.4, there exists an $M>0$ such that $\left|t_{k}\right| \leq k M$. Hence,

$$
\begin{aligned}
&\left|f(x)-\left(A_{\lambda} s\right)_{n}\right| \leq M \sum_{k=1}^{\infty} k \int_{x_{n}}^{x_{n+1}} t^{k-1}(1-t) d t \\
&=M \int_{x_{n}}^{x_{n+1}}(1-t) \sum_{k=1}^{\infty} k t^{k-1} d t \\
&=M \int_{x_{n}}^{x_{n+1}} \frac{1}{1-t} d t \\
&=-M\left(\log \left(1-x_{n+1}\right)-\log \left(1-x_{n}\right)\right) \\
&=-M\left(\log \left(\frac{1}{\lambda(n+1)}\right)-\log \left(\frac{1}{\lambda(n)}\right)\right) \\
&=M \log \left(\frac{\lambda(n+1)}{\lambda(n)}\right) \\
&=o(1) .
\end{aligned}
$$

Since $s$ is $A_{\lambda}$-summable to $L$, we see that $\lim _{x \rightarrow 1^{-}} f(x)=L$. That is, $s$ is Abel summable to $L$, and hence, $A_{\lambda}$ is equivalent to the Abel method for bounded sequences.

The next theorem presents an equivalence relationship between the discrete Abel means when $\lambda$ and $\mu$ are asymptotic. 
THEOREM 2.6. Let $\lambda$ and $\mu$ be strictly increasing sequences of real numbers such that $\lambda(0) \geq 1, \mu(0) \geq 1, \lambda(n) \rightarrow \infty, \mu(n) \rightarrow \infty$, and $\lim _{n \rightarrow \infty}(\mu(n) / \lambda(n))=1$. Then $A_{\lambda}$ is equivalent to $A_{\mu}$ for bounded sequences.

Proof. We proceed as in the proof of Theorem 2.5. Let $s$ be a bounded sequence and let $a$ be the sequence such that $s_{n}=\sum_{k=0}^{n} a_{k}$. Let $M(n):=\max \{\lambda(n), \mu(n)\}$, $m(n):=\min \{\lambda(n), \mu(n)\}, x_{n}:=1-1 / m(n)$, and $y_{n}:=1-1 / M(n)$. Then $0 \leq x_{n} \leq$ $y_{n}<1$ and for a given $n$,

$$
\begin{aligned}
\left|\left(A_{\mu} s\right)_{n}-\left(A_{\lambda} s\right)_{n}\right| & =\left|\frac{1}{\mu(n)} \sum_{k=0}^{\infty} s_{k}\left(1-\frac{1}{\mu(n)}\right)^{k}-\frac{1}{\lambda(n)} \sum_{k=0}^{\infty} s_{k}\left(1-\frac{1}{\lambda(n)}\right)^{k}\right| \\
& =\left|\frac{1}{M(n)} \sum_{k=0}^{\infty} s_{k}\left(1-\frac{1}{M(n)}\right)^{k}-\frac{1}{m(n)} \sum_{k=0}^{\infty} s_{k}\left(1-\frac{1}{m(n)}\right)^{k}\right| \\
& =\left|\sum_{k=0}^{\infty} a_{k} y_{n}{ }^{k}-\sum_{k=0}^{\infty} a_{k} x_{n}{ }^{k}\right| .
\end{aligned}
$$

By Lemma 2.3,

$$
\begin{aligned}
\left|\left(A_{\mu} s\right)_{n}-\left(A_{\lambda} s\right)_{n}\right| & =\left|\sum_{k=1}^{\infty} t_{k} \Delta\left(\frac{y_{n}^{k}}{k}\right)-\sum_{k=1}^{\infty} t_{k} \Delta\left(\frac{x_{n}^{k}}{k}\right)\right| \\
& =\left|\sum_{k=1}^{\infty} t_{k} \int_{x_{n}}^{y_{n}} t^{k-1}(1-t) d t\right| \\
& \leq \sum_{k=1}^{\infty}\left|t_{k}\right| \int_{x_{n}}^{y_{n}} t^{k-1}(1-t) d t .
\end{aligned}
$$

By Lemma 2.4, there exists an $M>0$ such that $\left|t_{k}\right| \leq k M$. Hence,

$$
\begin{aligned}
\left|\left(A_{\mu} s\right)_{n}-\left(A_{\lambda} s\right)_{n}\right| & \leq M \sum_{k=1}^{\infty} k \int_{x_{n}}^{y_{n}} t^{k-1}(1-t) d t \\
& =M \int_{x_{n}}^{y_{n}}(1-t) \sum_{k=1}^{\infty} k t^{k-1} d t \\
& =M \int_{x_{n}}^{y_{n}} \frac{1}{1-t} d t \\
& =-M\left(\log \left(1-y_{n}\right)-\log \left(1-x_{n}\right)\right) \\
& =-M\left(\log \left(\frac{1}{M(n)}\right)-\log \left(\frac{1}{m(n)}\right)\right) \\
& =M \log \left(\frac{M(n)}{m(n)}\right) \\
& =o(1),
\end{aligned}
$$

since $\lim _{n \rightarrow \infty}(M(n) / m(n))=\lim _{n \rightarrow \infty}(\mu(n) / \lambda(n))=1$. Hence, if $s$ is $A_{\lambda}$-summable to $L$, then

$$
0 \leq\left|\left(A_{\mu} s\right)_{n}-L\right| \leq\left|\left(A_{\mu} s\right)_{n}-\left(A_{\lambda} s\right)_{n}\right|+\left|\left(A_{\lambda} s\right)_{n}-L\right|=o(1)+o(1)=o(1) .
$$


Similarly, if $s$ is $A_{\mu}$-summable to $L$, then

$$
0 \leq\left|\left(A_{\lambda} s\right)_{n}-L\right| \leq\left|\left(A_{\lambda} s\right)_{n}-\left(A_{\mu} s\right)_{n}\right|+\left|\left(A_{\mu} s\right)_{n}-L\right|=o(1)+o(1)=o(1) .
$$

Thus, $A_{\lambda}$ and $A_{\mu}$ are equivalent for bounded sequences.

To see that $\lim _{n \rightarrow \infty}(\mu(n) / \lambda(n))=1$ is not a necessary condition in Theorem 2.6, simply consider the sequences $\lambda(n):=n^{2}$ and $\mu(n):=n^{3}$. Then

$$
\lim _{n \rightarrow \infty} \frac{\lambda(n+1)}{\lambda(n)}=\lim _{n \rightarrow \infty} \frac{\mu(n+1)}{\mu(n)}=1,
$$

and hence, by Theorem 2.5, $A_{\lambda}, A_{\mu}$, and the Abel method are all equivalent for bounded sequences. However, $\lambda$ and $\mu$ are not asymptotic.

\section{REFERENCES}

[1] D. H. Armitage and I. J. Maddox, Discrete Abel means, Analysis 10 (1990), no. 2-3, 177-186.

JefFrey A. Osikiewicz: Department of Mathematical SCIEnCEs, Kent State UniVersity, Tuscarawas Campus, 330 University DR NE, NeW PhiladelPhia, OH 44663-9403, USA

E-mail address: josikiewicz@tusc.kent.edu 


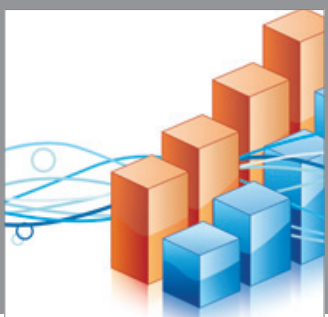

Advances in

Operations Research

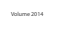

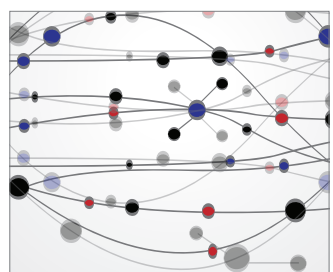

\section{The Scientific} World Journal
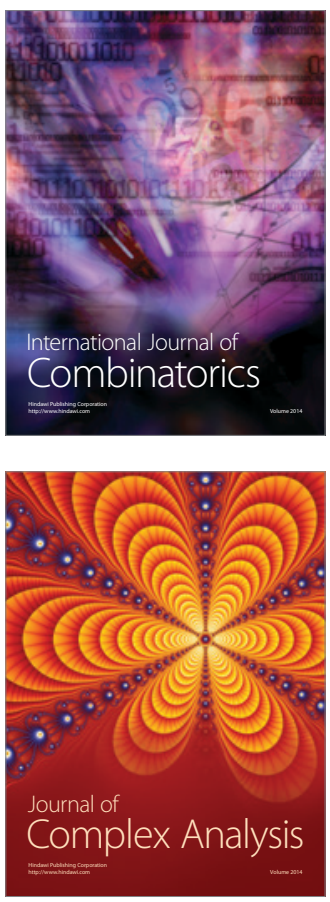

International Journal of

Mathematics and

Mathematical

Sciences
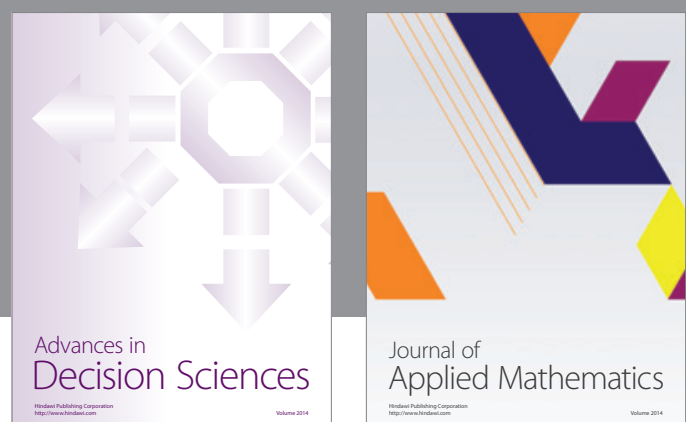

Journal of

Applied Mathematics
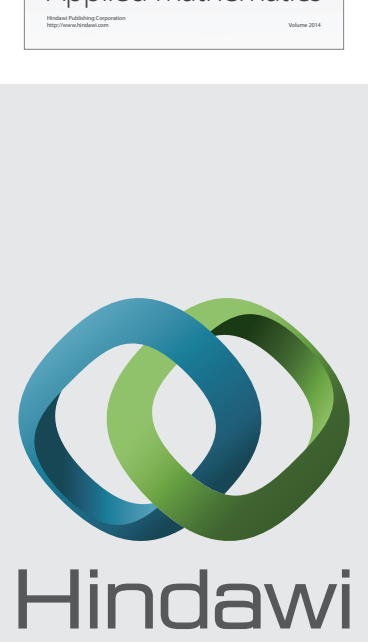

Submit your manuscripts at http://www.hindawi.com
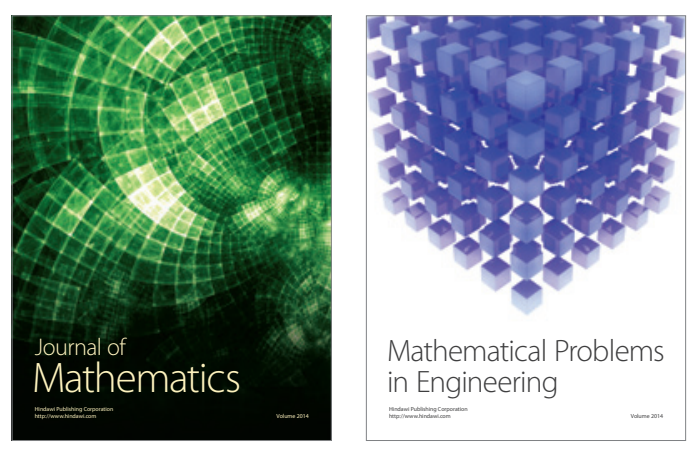

Mathematical Problems in Engineering
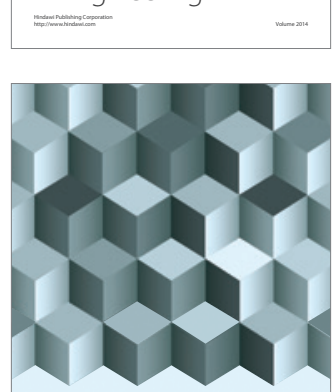

Journal of

Function Spaces
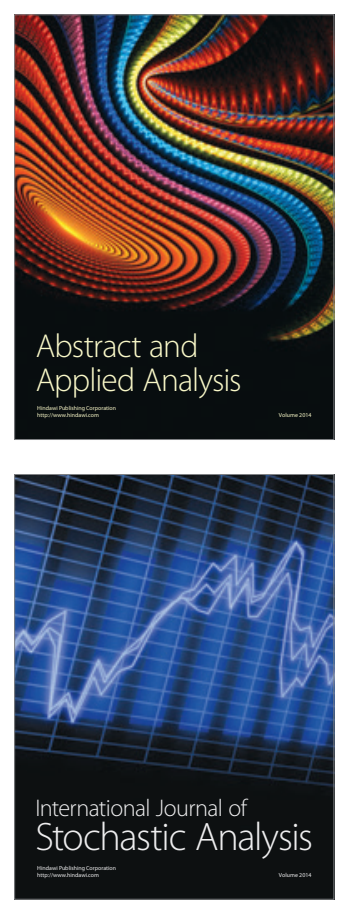

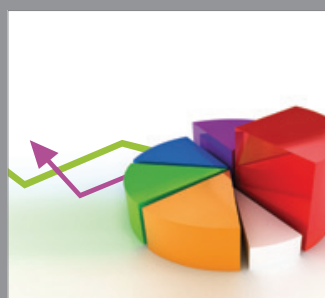

ournal of

Probability and Statistics

Promensencen
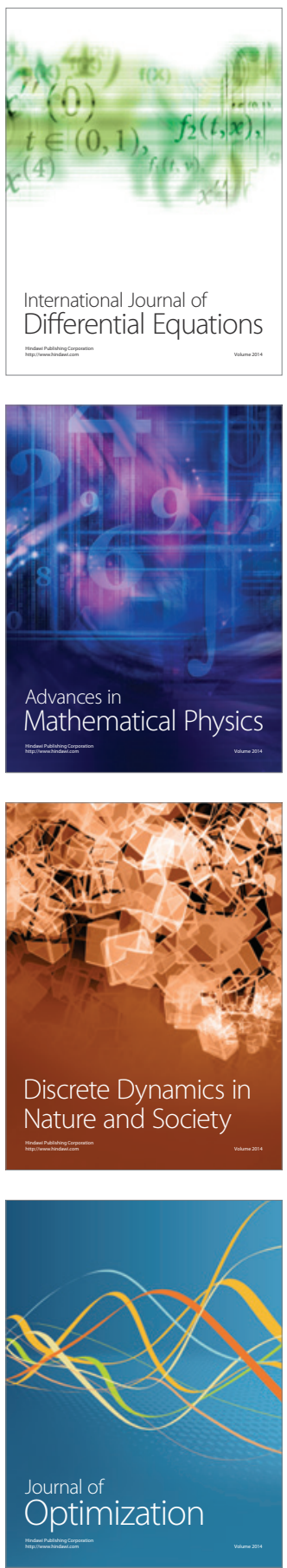